\title{
An expedient route for the reduction of carboxylic acids to alcohols employing 1-propanephosphonic acid cyclic anhydride as acid activator
}

\author{
G. Nagendra, C. Madhu, T. M. Vishwanatha, Vommina V. Sureshbabu* \\ \#109, Peptide Research Laboratory, Department of Studies in Chemistry, Central College Campus, Bangalore University, Dr. B. R. Ambedkar Veedhi, Bangalore 560 001, India
}

\section{A R T I C L E I N F O}

\section{Article history:}

Received 11 April 2012

Revised 25 June 2012

Accepted 26 June 2012

Available online 20 July 2012

\section{Keywords:}

1-Propanephosphonic acid cyclic anhydride (T3P)

Reduction

Aryl carboxylic acids

Alcohol

$\mathrm{N}^{\alpha}$-Protected amino acid

Peptide acid

$\beta / \gamma$-Aminols

Peptibols

\begin{abstract}
A B S T R A C T
A simple and efficient method for the synthesis of alcohols from the corresponding carboxylic acids is described. Activation of carboxylic acid with 1-propanephosphonic acid cyclic anhydride (T3P) and subsequent reduction using $\mathrm{NaBH}_{4}$ yield the alcohol in excellent yields with good purity. Reduction of several alkyl/aryl carboxylic acids and $\mathrm{N}^{\alpha}$-protected amino acids/peptide acids as well as $\mathrm{N}^{\beta}$-protected amino acids was successfully carried out to obtain corresponding alcohols in good yields. All the products were fully characterized by ${ }^{1} \mathrm{H}$ NMR and mass spectral analyses. The procedure is mild, simple and the isolation of the products is easy.
\end{abstract}

(c) 2012 Elsevier Ltd. All rights reserved.
Reduction of carboxylic acids, ketones, and aldehydes to alcohols is an important functional group transformation. Though there are several procedures known, more efficient and convenient protocols are continously being explored. The reduction of carboxylic acids was achieved by a variety of metal hydride based reducing agents such as $\mathrm{LiAlH}_{4}, \mathrm{AlH}_{3}$, sodium bis(2-methoxyethoxy)aluminum hydride, diisobutyl aluminum hydride, etc. ${ }^{1}$ Several borane reagent systems are also developed for the reduction of carboxyic acids. ${ }^{2}$ Reduction using $\mathrm{NaBH}_{4}$ in combination with $\mathrm{I}_{2},{ }^{3} \mathrm{ZrCl}_{4}{ }^{4}$, catecol-TFA, ${ }^{5} \mathrm{H}_{2} \mathrm{SO}_{4},{ }^{6} \mathrm{TiCl}_{4},{ }^{7} \mathrm{CaCl}_{2},{ }^{8}$ diglyme, ${ }^{9}$ and $\mathrm{Br}_{2}{ }^{10}$ were also reported in the literature. $\mathrm{BOP}$ reagent was used to activate carboxylic group prior to $\mathrm{NaBH}_{4}$ treatment. ${ }^{11}$ Cardenas group reported an elegant procedure for the reduction of caboxylic acids through the reaction of in situ formed hydroxybenzotriazole esters with $\mathrm{NaBH}_{4}$ in water. ${ }^{12}$

1-Propanephosphonic acid cyclic anhydride (T3P) is known as an efficient and reliable coupling agent and also a water scavenger. It has been employed for the conversion of carboxylic acids to aldehydes, amides to nitriles, and formamides to isonitriles. It has also been utilized in the synthesis of heterocycles, Weinreb amides, $\beta$-lactams, hydroxamic acids, acylazides, esters, imidazopyridines, dihydropyrimidinones as well as peptides (Fig. 1). ${ }^{13}$ T3P offers

\footnotetext{
* Corresponding author. Tel.: +91 082296 1339, +9199 86312937.

E-mail addresses: hariccb@hotmail.com, sureshbabuvommina@rediffmail.com (V.V. Sureshbabu).
}

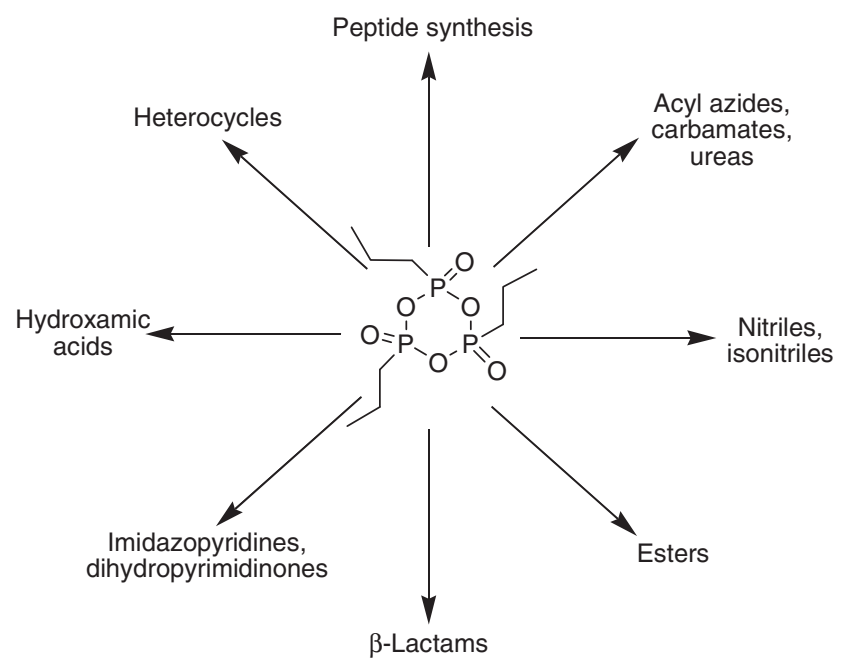

Figure 1. Applications of T3P in organic synthesis.

several advantages over traditional reagents such as solubility, ease of work-up, broad variety of functional group tolerance, low epimerization, and high yielding. Hence T3P is being explored in several organic reactions. ${ }^{14}$ Recently we reported an application of T3P as carboxylic acid activator in the synthesis of $\mathrm{N}^{\alpha}$-protected amino acid derived acyl azides and hydroxamic acids. ${ }^{15}$ Now we 


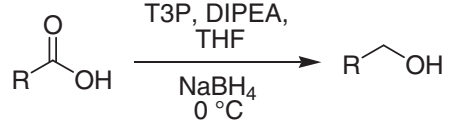

Scheme 1. General scheme for the reduction of carboxylic acids using T3P/ $\mathrm{NaBH}_{4}$.

herein report a simple approach for the synthesis of alcohols from corresponding carboxylic acids using $\mathrm{T} 3 \mathrm{P}-\mathrm{NaBH}_{4}$ reduction (Scheme 1).

Initially, the reduction of 4-methylbenzoic acid 1c was undertaken. 4-Methylbenzoic acid dissolved in THF was cooled to $0^{\circ} \mathrm{C}$. To this diisopropyl ethylamine (DIPEA) and 50\% T3P in ethyl acetate (EtOAc) were added and the reaction mixture was stirred for about 5 min. Then aqueous $\mathrm{NaBH}_{4}$ was added and the reaction was allowed to stir at the same temperature. After the complete consumption of 4-methylbenzoic acid 1c, as indicated by TLC (took about $30 \mathrm{~min}$ ), the solvent was evaporated and the crude compound was extracted into EtOAc. The organic phase was washed with $10 \% \mathrm{Na}_{2} \mathrm{CO}_{3}$ solution, water, and brine followed by evaporation of the solvent to yield (4-methylphenyl)methanol 2c in $89 \%$ yield. The efficacy of the protocol was further studied by reducing a series of aryl and alkyl carboxylic acids $\mathbf{1 a - 1 k}$ to their corresponding alcohols 2a-2k (Scheme 2, Table 1). All the products were isolated in good yields (above 80\%) and were well characterized. ${ }^{16,17}$

Further, the utility of T3P to reduce the $\mathrm{N}^{\alpha}$-protected amino acids/peptide acids $\mathbf{3 a}-\mathbf{3 n}$ to their corresponding alcohols $\mathbf{4 a - 4 n}$ was undertaken. The $\mathrm{N}^{\alpha}$-protected aminols/peptibols serve as precursors for the preparation of novel amino acid derived building blocks such as aldehydes, oxymethylene peptides, mesylates, and diamines. ${ }^{18,19}$ They have also been extensively used in asymmetric synthesis, ${ }^{20}$ in the synthesis of insecticidal compounds, ${ }^{21}$ reduced amide bonds, ${ }^{22}$ used as intermediates in the synthesis of ureidopeptides, vicinal diamines, ${ }^{23}$ and peptidosulfonamides. ${ }^{24}$ In addition, $\beta$-amino alcohols serve as precursors for the synthesis of $\mathrm{N}$ protected amino alkyl $p$-nitrophenyl carbonates and $N$-Fmoc- $\beta$ amino alkoxy carbonyl chlorides which are the building blocks in the construction of oligopeptidyl carbamates. ${ }^{25}$ In general, the $\mathrm{N}^{\alpha}$-protected aminols/peptibols were prepared by borane mediated reduction of $\mathrm{N}$-protected amino acids. ${ }^{18 \mathrm{~b}}$ The reduction was carried out employing activated carboxylic acid derivatives such as mixed anhydrides, ${ }^{26}$ acid halides, ${ }^{27}$ acyl benzotriazoles, ${ }^{28} \mathrm{~N}$-carboxy anhydrides (NCA's) ${ }^{29}$ active esters ${ }^{30}$, and $\mathrm{NaBH}_{4}$. Reaction of $\mathrm{N}$-protected amino acids with cyanuric chloride and $\mathrm{NaBH}_{4}$ was also documented. ${ }^{31}$ In the present work, we started our initial study by reducing $N^{\alpha}$-Fmoc-Phe-OH $3 \mathbf{a}$ to the corresponding aminol 4a. For this, initially $N^{\alpha}$-Fmoc-Phe-OH 3a was dissolved in THF and was treated with DIPEA and 50\% T3P in EtOAc at $0{ }^{\circ} \mathrm{C}$ and the reaction mixture was stirred for $5 \mathrm{~min}$. Then aqueous $\mathrm{NaBH}_{4}$ was added to the reaction mixture and the stirring was continued at the same temperature till the complete consumption of the starting material (as indicated by TLC analysis). A simple work-up led to the isolation of Fmoc-Phe-ol $\mathbf{4 a}$ in $96 \%$ yield and the purity of the crude product was found to be $99 \%$ as determined by RPHPLC $^{35}$ analysis (Fig. 2). Following successful isolation of product 4a, several $\mathrm{N}^{\alpha}$-protected amino acids/peptide acids $\mathbf{3 b} \mathbf{b} \mathbf{3 n}$ were
Table 1

Reduction of aryl and alkyl carboxylic acids to corresponding alcohols

\begin{tabular}{|c|c|c|c|c|}
\hline Entry & Alcohol & Time (min) & $\operatorname{Mp}\left({ }^{\circ} \mathrm{C}\right)$ & Yield $^{\mathrm{a}}(\%)$ \\
\hline $2 a$ & & 25 & Liq. & 83 \\
\hline $2 \mathbf{b}$ & & 25 & $91-93$ & 91 \\
\hline 2c & & 30 & $58-60$ & 89 \\
\hline 2d & & 28 & Liq. & 90 \\
\hline $2 \mathbf{e}$ & & 30 & Liq. & 80 \\
\hline $2 f$ & & 30 & $108-110$ & $86^{\mathrm{b}}$ \\
\hline $2 \mathrm{~g}$ & & 22 & Liq. & 93 \\
\hline $2 \mathbf{h}$ & & 18 & $113-115$ & 89 \\
\hline $\mathbf{2 i}$ & & 20 & Liq. & 90 \\
\hline $2 \mathbf{j}$ & & 22 & Liq. & 92 \\
\hline 2k & & 28 & Semi solid & $84^{\mathrm{b}}$ \\
\hline
\end{tabular}

Isolated yields.

b 2.2 equiv of DIPEA, 4.0 equiv of 50\% T3P in EtOAc, and 4.0 equiv of $\mathrm{NaBH}_{4}$ were used.

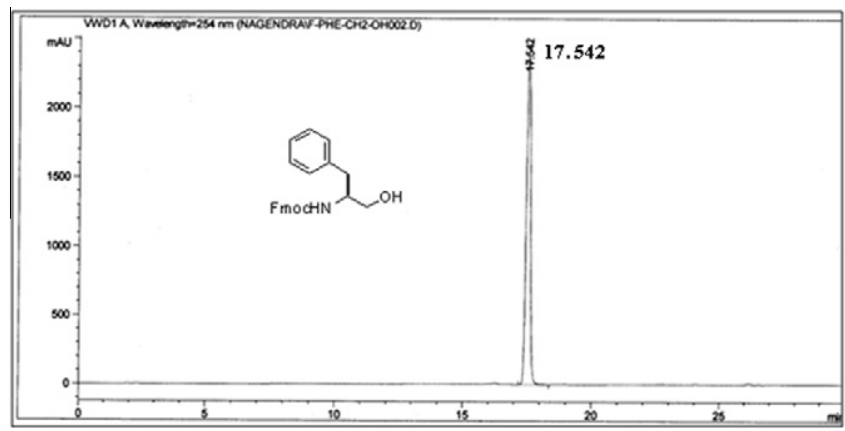

Figure 2. Crude RP-HPLC profile of $N^{\alpha}$-Fmoc-Phe-ol 4a.

then reduced to their corresponding alcohols $\mathbf{4 b} \mathbf{- 4 n}$ which were isolated in good yields (above 80\%). All the compounds prepared

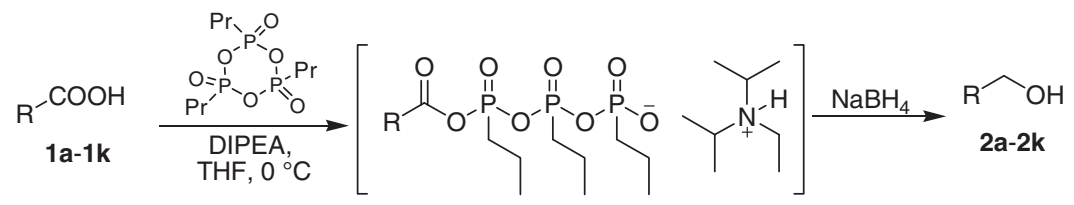

$\mathrm{R}=$ aryl or alkyl group

Scheme 2. Reduction of carboxylic acids to corresponding alcohols mediated by T3P. 
<smiles>[R16]NC([R7])CO</smiles>

Scheme 3. Reduction of $\mathrm{N}^{\alpha}$-protected amino acid/peptide acids to corresponding aminols/peptibols. were characterized by NMR and mass spectroscopy (Scheme 3, Table 2). ${ }^{16,32}$

The $\gamma$-aminols serve as useful precursors in the synthesis of $\beta$-amino aldehydes which are used in the construction of $\alpha / \beta$ unsaturated peptidomimetics as well as in the synthesis of several heterocycles. The amino aldehydes can be obtained through the IBX mediated oxidation of $\mathrm{N}$-protected $\beta$-aminols. ${ }^{33}$ In the present work, we prepared few $N$-Fmoc/Boc and Z-protected $\gamma$-aminols

Table 2

Reduction of $\mathrm{N}^{\alpha}$-protected amino acids/peptide acids to corresponding alcohols

\begin{tabular}{|c|c|c|c|c|}
\hline Entry & Alcohol & Time (min) & $\mathrm{Mp}\left({ }^{\circ} \mathrm{C}\right)$ & Yield (\%) \\
\hline $4 a$ & & 25 & $130-132$ & 96 \\
\hline $4 b$ & & 25 & $120-122$ & 88 \\
\hline $4 c$ & & 28 & $107-109$ & 85 \\
\hline 4d & & 24 & $128-130$ & 91 \\
\hline $4 e$ & & 22 & $55-57$ & 90 \\
\hline $4 f$ & & 25 & $86-88$ & 92 \\
\hline $4 g$ & & 28 & $61-63$ & 88 \\
\hline $4 h$ & & 35 & liq. & 82 \\
\hline $4 i$ & & 35 & liq. & 84 \\
\hline $4 j$ & & 35 & $180-182$ & 88 \\
\hline $4 k$ & & 40 & $108-110$ & 80 \\
\hline 41 & & 36 & $141-143$ & 91 \\
\hline $4 \mathrm{~m}$ & BocHN & 32 & $\mathrm{nd}^{\mathrm{a}}$ & 86 \\
\hline $4 n$ & & 42 & $78-80$ & 88 \\
\hline
\end{tabular}

a nd: not determined. 


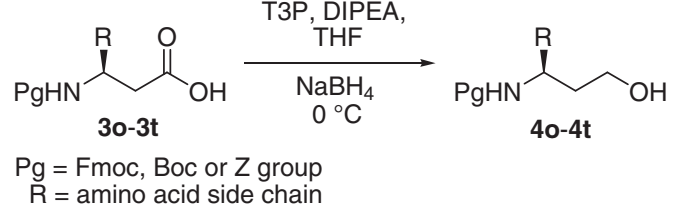

Scheme 4. Reduction of $\mathrm{N}^{\beta}$-protected amino acids to corresponding $\gamma$-amino alcohols.

Table 3

Reduction of $\mathrm{N}^{\beta}$-protected amino acids to corresponding alcohols

\begin{tabular}{|c|c|c|c|}
\hline$\beta$-Amino acid, 3 & $\gamma$-Amino alcohol, 4 & $\operatorname{Mp}\left({ }^{\circ} \mathrm{C}\right)$ & Yield (\%) \\
\hline Fmoc- $\beta$-Ala-OH, $3 \mathbf{o}$ & Fmoc- $\gamma$-Ala-ol, $\mathbf{4 o}$ & $129-131$ & 96 \\
\hline Fmoc- $\beta-\operatorname{Lys}(Z)-O H, 3 p$ & Fmoc- $\gamma$-Lys(Z)-ol, $\mathbf{4 p}$ & $95-97$ & 84 \\
\hline Fmoc- $\beta$-Asp(Bzl)-OH, $\mathbf{3 q}$ & Fmoc- $\gamma$-Asp(Bzl)-ol, $\mathbf{4 q}$ & $122-124$ & 91 \\
\hline Boc- $\beta-$ Val-OH, $\mathbf{3 r}$ & Boc- $\gamma$-Val-ol, $\mathbf{4 r}$ & $44-46$ & 89 \\
\hline Boc- $\beta$-Leu-OH, $3 \mathbf{s}$ & Boc- $\gamma$-Leu-ol, $4 s$ & $64-66$ & 90 \\
\hline Z- $\beta$-Phe-OH, 3t & Z- $\gamma$-Phe-ol, 4t & $\mathrm{nd}^{\mathrm{a}}$ & 88 \\
\hline
\end{tabular}

a nd: not determined.

through $\mathrm{T} 3 \mathrm{P}-\mathrm{NaBH}_{4}$ mediated reduction of corresponding N-protected $\beta$-amino acids (Scheme 4 ). The results are summarized in Table 3. ${ }^{16,34}$

During this study, we also synthesized a set of enantiomeric aminols $\mathrm{N}$-Fmoc-L-Ala-ol $\mathbf{4 b}$, and $\mathrm{N}$-Fmoc-D-Ala-ol $\mathbf{4} \mathbf{b}^{*}$ through the present protocol. The synthesis of aminols using T3P was found to be racemization-free as indicated by its chiral-HPLC profile. In the chiral-HPLC profile a single peak was observed at $R_{\mathrm{t}}$ $12.18 \mathrm{~min}$ for compound $\mathbf{4 b}$, and for its epimer $\mathbf{4} \mathbf{b}^{*}$, the $R_{\mathrm{t}}$ value was observed at $18.73 \mathrm{~min}^{36}$ (Fig. 3). This clearly confirms that the T3P mediated reduction of $\mathrm{N}^{\alpha}$-protected amino acids was found to be free from racemization.

In summary, the facile synthesis of alcohols by the reduction of corresponding carboxylic acids using T3P- $\mathrm{NaBH}_{4}$ is described. The protocol is simple, fast, and efficient for the reduction of both alkyl/ aryl aromatic carboxylic acids and $\mathrm{N}^{\alpha}$-protected amino acids/peptide acids. The isolation of the alcohol was easy and devoid of the pre-filtration step prior to $\mathrm{NaBH}_{4}$ addition. The protocol furnishes high yields of alcohols.

\section{Acknowledgments}

We thank CSIR (Grant No. 01(2323)/09/EMR-II, 2009) New Delhi, India for financial support. We also thank Archimica $\mathrm{GmbH}$,
Industrial park, Hoschst Building D56965926, Frankfurt am Main, Germany for providing 50\% T3P in EtOAc as gift sample.

\section{Supplementary data}

Supplementary data associated with this article can be found, in the online version, at http://dx.doi.org/10.1016/j.tetlet.2012.06. 108.

\section{References and notes}

1. (a) Karrer, P.; Suter, M. Helv. Chim. Acta 1948, 32, 1156; (b) Ito, A.; Takahashi, R. Baba, Y. Chem. Pharm. Bull. 1975, 23, 3081; (c) Brown, H. C. Boranes in Organic Chemistry; Cornell University Press: Ithaca, New York, 1971; Chapter 13.

2. (a) Yoon, N. M.; Pak, C. S.; Brown, H. C.; Krishnamurthy, S.; Stocky, T. P. J. Org. Chem. 1973, 38, 2786; (b) Brown, H. C.; Stocky, T. P. J. Am. Chem. Soc. 1977, 99 8218; (c) Chen, M. H.; Iakovleva, E.; Kasten, S.; Magano, J.; Rodriguez, D.; Sexton, K. E.; Zhang, J.; Lee, H. Org. Prep. Proceed. Int. 2002, 34, 665; (d) Pridgen, L. N.; Prol, J., Jr.; Alexander, B.; Gillyard, L. J. Org. Chem. 1989, 54, 3231; (e) Cho, S.-D.; Park, Y.-D.; Kim, J.-J.; Falck, J. R.; Yoon, Y.-J. Bull. Korean Chem. Soc. 2004 $25,407$.

3. (a) Bhaskar Kanth, J. V.; Periasamy, M. J. Org. Chem. 1991, 56, 5964; (b) McKennon, M.; Meyers, A. I. J. Org. Chem. 1993, 58, 3568; (c) Simek, J. W.; Tuck, T.; Bush, K. C. J. Chem. Edu. 1997, 74, 107.

4. Itsuno, S.; Sakarai, Y.; Ito, K. Synthesis 1988, 995.

5. Suseela, Y.; Periasamy, M. Tetrahedron 1992, 48, 371.

6. Abiko, A.; Maamune, S. Tetrahedron Lett. 1992, 33, 5517.

7. Kano, S.; Tanaka, Y.; Sugino, E.; Hibino, S. Synthesis 1980, 695.

8. Yan, S.-Q.; Suyi, H.-Z.; Zhang, Y.-L. Gansu Sci. 1996, 8, 71.

9. (a) Yang, C.; Pittman, C. U., Jr. Synth. Commun. 1998, 28, 2027; (b) Hua-Jie, Z.; Pittman, C. U., Jr. Synth. Commun. 2003, 33, 1733.

10. Tudge, M.; Mashima, H.; Savarin, C.; Humphrey, G.; Davies, I. Tetrahedron Lett 2008, 49, 1041.

11. McGeary, R. P. Tetrahedron Lett. 1998, 39, 3319-3322.

12. Morales-Serna, J. A.; Garcia-Rios, E.; Bernal, J.; Paleo, E.; Gavino, R.; Cardenas, J. Synthesis 2011, 1375. and the references sited therein.

13. (a) Llanes, A. L. Synlett 2007, 1328. and references cited therein; (b) Augustine J. K.; Atta, R. N.; Ramappa, B. K.; Boodappa, C. Synlett 2009, 3378; (c) Meudt, A.; Scherer, S.; Böhm, C. PCT Int. Appl. WO 2005102978, 2005; Chem. Abstr. 2005, 143, 440908.; (d) Escher, R.; Buning, P. Angew. Chem., Int. Ed. Engl. 1986, 25, 277; (e) Klose, J.; Bienert, M.; Mollenkopf, C.; Wehle, D.; Zhang, C.-W.; Carpino, L. A.; Henklein, P. Chem. Commun. 1847, 1999; (f) Zumpe, F. L.; Flub, M.; Schmitz, K.; Lender, A. Tetrahedron Lett. 2007, 48, 1421; (g) Meudt, A.; Scherer, S.; Bohm, C. PCT Int. Appl. WO 2005123632, 2005.; (h) Kessler, K. Angew. Chem., Int. Ed. Engl. 1997, 36, 1191; (i) Jida, M.; Deprez, B. New J. Chem. 2012, 36, 869873.

14. Jida, M.; Deprez, B. New J. Chem 2012, 36, 869.

15. (a) Basavaprabhu; Narendra, N.; Lamani, R. S.; Sureshbabu, V. V. Tetrahedron Lett. 2010, 51, 3002; (b) Vasantha, B.; Hemantha, H. P.; Sureshbabu, V. V. Synthesis 2010, 2990.

16. General procedure for the reduction of carboxylic acids to alcohols using T3P$\mathrm{NaBH}_{4}$ : To a solution of carboxylic acid $(10 \mathrm{mmol})$ in THF $(10 \mathrm{~mL})$, DIPEA $(11 \mathrm{mmol}, 1.42 \mathrm{~mL})$ and $50 \% \mathrm{~T} 3 \mathrm{P}$ in EtOAc $(20 \mathrm{mmol}, 6.36 \mathrm{~mL})$ were added at $0{ }^{\circ} \mathrm{C}$ and the solution was stirred for about $10 \mathrm{~min}$. Then aqueous solution of $\mathrm{NaBH}_{4}\left(10 \mathrm{mmol}, 388 \mathrm{mg}\right.$ in $0.3 \mathrm{~mL}$ of $\left.\mathrm{H}_{2} \mathrm{O}\right)$ was added to the reaction mixture at the same temperature and the reaction was allowed to stir till the completion of the reaction as indicated by TLC. After the completion of the

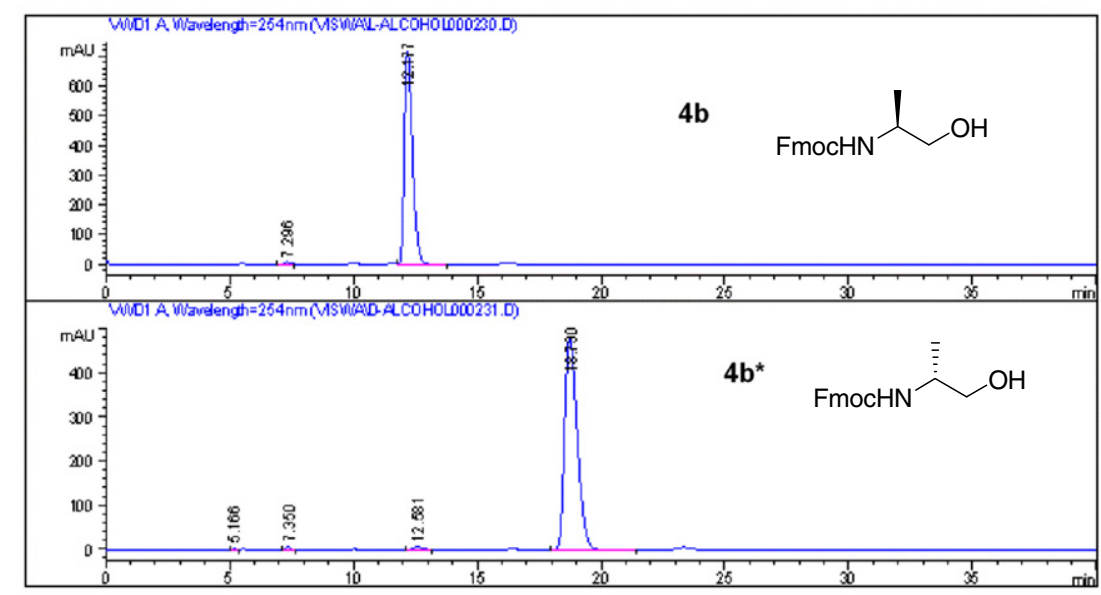

Figure 3. Racemization studies. 
reaction, the solvent was evaporated and the crude alcohol was extracted into EtOAc and the organic phase was washed with $5 \%$ citric acid $(10 \mathrm{~mL} \times 2), 5 \%$ $\mathrm{Na}_{2} \mathrm{CO}_{3}(10 \mathrm{~mL} \times 2)$, water, and brine solution. The product was isolated after the evaporation of solvent under reduced pressure and dried over anhydrous $\mathrm{Na}_{2} \mathrm{SO}_{4}$

17. Spectral data for p-tolylmethanol, 2c: Yield $89 \%$; $\mathrm{mp} 58-60^{\circ} \mathrm{C}$. TLC $\mathrm{R}_{f} 0.7$ (EtOAc:n-hexane, 1:9). RP-HPLC $R_{\mathrm{t}} 21.98 \mathrm{~min} .{ }^{1} \mathrm{H}$ NMR $\left(400 \mathrm{MHz}, \mathrm{CDCl}_{3}\right)$ : $\delta=2.22(\mathrm{~s}, 3 \mathrm{H}), 4.86(\mathrm{~s}, 2 \mathrm{H}), 5.31(\mathrm{t}, 1 \mathrm{H}), 6.91(\mathrm{~d}, 2 \mathrm{H}), 7.05(\mathrm{~d}, 2 \mathrm{H}) \mathrm{ppm} .{ }^{13} \mathrm{C}$ NMR $\left(100 \mathrm{MHz}, \mathrm{CDCl}_{3}\right): \delta=23.1,67.8,126.8,129.1,138.0,138.5 \mathrm{ppm}$. ESI-MS Calcd for $\mathrm{C}_{8} \mathrm{H}_{10} \mathrm{O} \mathrm{m} / \mathrm{z} 123.1[\mathrm{M}+\mathrm{H}]^{+}$. Found 123.0.

18. (a) Stanfield, C. F.; Parker, J. E.; Kanellis, P. J. Org. Chem. 1981, 46, 4797; (b) Thompson, R. C. Methods Enzymol. 1977, 46, 220; (c) Sharma, R. P.; Gore, M. G.; Akhtar, M. J. Chem. Soc., Chem. Commun. 1979, 875; (d) Ten Brink, R. E. J. Org Chem. 1987, 52, 418; (d) Pless, J.; Bauer, W.; Cardenaux, F.; Closse, A.; Hauser D.; Huguenia, R.; Roemer, D.; Buescher, H.-H.; Hill, R. C. Helv. Chim. Acta 1979, 62, 398; (e) Shang, L.; Wang, Q.; Fang, H.; Mu, J.; Wang, X.; Yuan, Y.; Wang, B.; Xu, W. Bioorg. Med. Chem. 2008, 16, 9984; (f) Kokotos, G.; Constantiou-Kokotou, V. J. Chem. Res. (S) 1992, 391, 3117.

19. (a) Cassal, J-M; Furst, A. Meier, W. Helv Chim Acta 1976, 59, 1917; (b) Thaisrivongs, S.; Pals, D. T.; Kati, W. M.; Turner, S. R.; Thosmosco, L. M.; Watt, W. J. Med. Chem. 1986, 29, 2080.

20. (a) Bolm, C. Angew. Chem., Int. Ed. 1991, 30, 542; (b) Coppola, G. M.; Schuster, H. F. Asymmetric Synthesis; John Wiley and Sons: New York, USA, 1987. 85.

21. Wu, S.; Takeya, R.; Eto, M.; Tomizawac J. Pestic Sci. 1987, 12, 221.

22. Martinez, J.; Bali, J. P.; Rodriguez, M.; Castro, B.; Magous, R.; Laur, J.; Lignon, M. F. J. Med. Chem. 1985, 28, 1874.

23. (a) Boeijen, A.; Ameijde, J. V.; Liskamp, R. M. J. J. Org. Chem. 2001, 66, 8454; (b) Guichard, G.; Semetey, V.; Didierjean, C.; Aubry, A.; Briand, J. P.; Rodriguez, M.J. Org. Chem. 1999, 64, 8702.

24. Monnee, M. C. F.; Marijne, M. F.; Brouwer, A. J.; Liskamp, R. M. J. Tetrahedron Lett. 2000, 41, 7991.

25. (a) Cho, C. Y.; Morna, E. J.; Cherry, S. R.; Stephans, J. C.; Fodor, S. P.; Adams, C. L.; Sundaram, A.; Jacobs, J. W.; Schultz, P. G. Science 1993, 261, 1303; (b) Sureshbabu, V. V.; Sudarshan, N. S.; Venkataramanarao, R. J. Pept. Res. Ther. 2008, 14, 149; (c) Cho, C. Y.; Youngquist, R. S.; Paikoff, S. J.; Baresini, M. H.; Hebert, A. R.; Berleau, L. T.; Liu, C. W.; Wemmer, D. E.; Keough, T.; Schultz, P. G. J. Am. Chem. Soc. 1998, 120, 7706.

26. (a) Kokotos, G. Synthesis 1990, 299; (b) Rodriguez, M.; Llinares, M.; Doulut, S.; Heitz, A.; Martinez, J. Tetrahedron Lett. 1991, 32, 923.
27. Kokotos, G.; Noula, C. J. Org. Chem. 1996, 61, 6994

28. Sureshbabu, V. V.; Sudarshan, N. S.; Muralidhar, L.; Narendra, N. J. Chem. Res. 2007, 683.

29. Fehrentz, J.-A.; Califano, J.-C.; Amblard, M.; Lottet, A.; Martinez, J. Tetrahedron Lett. 1994, 35, 569 .

30. (a) Nikawa, J.; Shiba, T. Chem. Lett. 1979, 981; (b) Jadhav, S. V.; Bandyopadhyay, A.; Benke, S. N.; Mali, S. M.; Gopi, H. N. Org. Biomol. Chem. 2011, 9, 4182; (c) Papavassilopoulou, E.; Christofis, P.; Terzoglou, D.; Moutevelis-Minakakis, P. Tetrahedron Lett. 2007, 48, 8323-8325.

31. Falorni, M.; Porcheddu, A.; Taddei, M. Tetrahedron Lett. 1999, 40, 4395-4396.

32. Spectral data for (S)-(9H-fluoren-9-yl)methyl 1-hydroxypropan-2-ylcarbamate, (Fmoc-L-Ala-ol) 4b: Yield 88\%; mp $120-122^{\circ} \mathrm{C}$. TLC $R_{\mathrm{f}} 0.5$ (EtOAc:n-hexane, 2:8). RP-HPLC $R_{\mathrm{t}} 12.39 \mathrm{~min} .{ }^{1} \mathrm{H}$ NMR (400 MHz, DMSO- $\left.d_{6}\right): \delta=1.02(\mathrm{~d}, 3 \mathrm{H})$, 3.17-3.21 (m, 1H), $3.31(\mathrm{~m}, 1 \mathrm{H}), 3.45-3.51(\mathrm{~m}, 1 \mathrm{H}), 4.19(\mathrm{t}, J=6.3 \mathrm{~Hz}, 1 \mathrm{H}), 4.26$ $(\mathrm{d}, J=7.4 \mathrm{~Hz}, 2 \mathrm{H}), 4.63(\mathrm{t}, J=5.7 \mathrm{~Hz}, 1 \mathrm{H}), 7.04(\mathrm{~d}, J=7.8 \mathrm{~Hz}, 1 \mathrm{H}), 7.32(\mathrm{t}$, $J=7.4 \mathrm{~Hz}, 2 \mathrm{H}), 7.40(\mathrm{t}, J=7.2 \mathrm{~Hz}, 2 \mathrm{H}), 7.69(\mathrm{~d}, J=7.4 \mathrm{~Hz}, 2 \mathrm{H}), 7.88(\mathrm{~d}, J=7.4 \mathrm{~Hz}$ 2H) ppm. ${ }^{13} \mathrm{C}$ NMR $\left(100 \mathrm{MHz}\right.$, DMSO- $\left.d_{6}\right): \delta=17.29,46.74,48.43,64.47,65.14$, $120.08,125.18,127.02,127.57,140.71,143.94,155.57$ ppm. HRMS Calcd for $\mathrm{C}_{18} \mathrm{H}_{19} \mathrm{NO}_{3} \mathrm{~m} / \mathrm{z} 320.1263[\mathrm{M}+\mathrm{Na}]^{+}$. Found 320.1261 .

33. Chen, J. J.; Aduda, V. Synth. Commun. 2007, 37, 3493.

34. Spectral data for (S)-(9H-fluoren-9-yl)methyl 4-hydroxybutan-2-ylcarbamate, (Fmoc- $\gamma$-Ala-ol) 4o: Yield 96\%; mp 129-131 ${ }^{\circ} \mathrm{C}$. TLC $R_{\mathrm{f}} 0.3$ (EtOAc/n-hexane, 3:7). RP-HPLC $R_{\mathrm{t}} 17.69 \mathrm{~min} .{ }^{1} \mathrm{H}$ NMR $\left(400 \mathrm{MHz}\right.$, DMSO- $\left.d_{6}\right): \delta=1.24$ (d, $J=6.8 \mathrm{~Hz}, 3 \mathrm{H}), 1.55(\mathrm{~m}, 2 \mathrm{H}), 3.41(\mathrm{~m}, 2 \mathrm{H}), 3.73(\mathrm{~m}, 1 \mathrm{H}), 4.11(\mathrm{t}, J=6.0 \mathrm{~Hz}$, $1 \mathrm{H}), 4.14-4.24(\mathrm{~m}, 3 \mathrm{H}), 5.18(\mathrm{br}, 1 \mathrm{H}), 7.28(\mathrm{t}, J=7.2 \mathrm{~Hz}, 2 \mathrm{H}), 7.36(\mathrm{t}, J=7.6 \mathrm{~Hz}$, $2 \mathrm{H}), 7.61(\mathrm{~d}, J=5.6 \mathrm{~Hz}, 2 \mathrm{H}), 7.84(\mathrm{~d}, J=7.2 \mathrm{~Hz}, 2 \mathrm{H}) \mathrm{ppm} .{ }^{13} \mathrm{C} \mathrm{NMR}(100 \mathrm{MHz}$, DMSO- $\left.d_{6}\right): \delta=21.13,37.67,41.13,47.27,58.38,65.57,120.54,125.69,127.49$, $128.50,141.19,144.38,156.11 \mathrm{ppm}$. HRMS Calcd for $\mathrm{C}_{19} \mathrm{H}_{21} \mathrm{NO}_{3} \mathrm{~m} / z 334.1419$ $[\mathrm{M}+\mathrm{Na}]^{+}$. Found 334.1422 .

35. HPLC particulars: Agilent 1100 series having G1311A VWD at $\lambda=254 \mathrm{~nm}$, flow $0.5 \mathrm{~mL} / \mathrm{min}$, column: agilent eclipse XDB-C18, pore size- $5 \mu \mathrm{m}$, diameter $\times$ length $=4.6 \times 150 \mathrm{~mm}$; method: gradient $0.1 \%$ TFA wateracetonitrile; acetonitrile $30-100 \%$ in $30 \mathrm{~min}$.

36. Chiral HPLC particulars: Agilent 1100 series having G1311A VWD at $\lambda=254 \mathrm{~nm}$, flow $1.0 \mathrm{~mL} / \mathrm{min}$, column: phenominex made Lux, pore size- $5 \mu$, Cellusole-1, diameter $\times$ length $=250 \times 4.6 \mathrm{~mm}$; method: $80: 20 n$-hexane $/$ isopropanol in isocratic mode in $40 \mathrm{~min}$. 